\title{
A contemporary survival analysis of individuals with cystic fibrosis: a cohort study
}

\author{
Anne L. Stephenson ${ }^{1-4}$, Melissa Tom ${ }^{2}$, Yves Berthiaume ${ }^{5}$, Lianne G. Singer ${ }^{4,6}$, \\ Shawn D. Aaron ${ }^{7}$, G.A. Whitmore ${ }^{7,8}$ and Sanja Stanojevic ${ }^{3,9}$ \\ Affiliations: ${ }^{1}$ Adult CF Program, St Michael's Hospital, Toronto, ON, Canada. ${ }^{2}$ Keenan Research Centre in the \\ $\mathrm{Li}$ Ka Shing Knowledge Institute of St Michael's Hospital, Toronto, ON, Canada. ${ }^{3}$ Research Institute, The \\ Hospital for Sick Children, Toronto, ON, Canada. ${ }^{4}$ Dept of Medicine, University of Toronto, Toronto, ON, \\ Canada. ${ }^{5} \mathrm{IRCM}$ and CHUM Université de Montréal, Montreal, QC, Canada. ${ }^{6}$ Toronto Lung Transplant Program, \\ University Health Network, Toronto, ON, Canada. ${ }^{7}$ The Ottawa Hospital Research Institute, University of \\ Ottawa, Ottawa, ON, Canada. ${ }^{8}$ Desautels Faculty of Management, McGill University, Montreal, QC, Canada. \\ ${ }^{9}$ Institute of Health Policy, Management and Evaluation, University of Toronto, Toronto, ON, Canada.
}

Correspondence: Anne Stephenson, St Michael's Hospital, 30 Bond Street, 6th floor, Bond Wing, Toronto, ON, M5B 1W8, Canada. E-mail: stephensonadsmh.ca

ABSTRACT Previously established predictors of survival may no longer apply in the current era of cystic fibrosis (CF) care. Our objective was to identify risk factors associated with survival in a contemporary CF population.

We used the Canadian CF Registry, a population-based cohort, to calculate median age of survival and summarise patient characteristics from 1990 to 2012. Clinical, demographic and geographical factors, and survival were estimated for a contemporary cohort (2000-2012) using Cox proportional hazards models.

There were 5787 individuals in the registry between 1990 and 2012. Median survival age increased from 31.9 years (95\% CI 28.3-35.2 years) in 1990 to 49.7 years (95\% CI 46.1-52.2 years) in the most current 5year window ending in 2012. Median forced expiratory volume in $1 \mathrm{~s}$ improved $(\mathrm{p}=0.04)$ and fewer subjects were malnourished $(\mathrm{p}<0.001)$ over time. Malnourished patients (hazard ratio (HR) $2.1,95 \% \mathrm{CI}$ 1.6-2.8), those with multiple exacerbations (HR 4.5, 95\% CI 3.2-6.4) and women with CF-related diabetes (HR 1.8, 95\% CI 1.2-2.7) were at increased risk of death.

Life expectancy in Canadians with CF is increasing. Modifiable risk factors such as malnutrition and pulmonary exacerbations are associated with an increased risk of death. The sex gap in CF survival may be explained by an increased hazard for death in women with CF-related diabetes.

@ERSpublications

Malnutrition and pulmonary exacerbations increase the risk of death in CF but survival in CF has increased over time http://ow.ly/CUqA9

Received: June 302014 | Accepted after revision: Oct 072014 | First published online: Nov 132014

Support statement: This study was supported by the Canadian Institute of Health Research. The study sponsor did not participate in the design or conduct of the study; collection, management, analysis or interpretation of the data; preparation, review or approval of the manuscript; and decision to submit the paper for publication.

Conflict of interest: None declared.

Copyright OERS 2015 


\section{Introduction}

Median age of survival in cystic fibrosis (CF) has increased in several countries $[1,2]$. Factors associated with poor survival include female sex, malnutrition, low lung function, certain infections and CF-related diabetes (CFRD) [3-9]. The availability of new inhaled antibiotics, Pseudomonas aeruginosa eradication therapy, mucolytic treatment, lung transplantation and, more recently, CF transmembrane conductance regulator (CFTR) modulators have inevitably had, or will have, an impact on survival in CF [10-13]. Furthermore, screening for complications has allowed early implementation of therapy and improved health [14]. Finally, newborn screening is now available in seven out of 10 provinces in Canada. With these advances in treatment and management, previously published survival models may no longer be applicable in the current era of CF care.

There is limited contemporary literature addressing the impact of demographic characteristics, clinical factors and CF-related complications on survival across the spectrum of CF disease severity. Two recent papers evaluating survival focused on specific subgroups. GEORGE et al. [15] studied individuals with severe lung disease (forced expiratory volume in $1 \mathrm{~s}$ (FEV1) $<30 \%$ predicted) and demonstrated that $50 \%$ of individuals with lung function below this range lived for an average of 5.3 years, much longer than the 2 years reported by Kerem et al. [3] in 1992. In addition, the study by GeORGE et al. [15] found malnutrition, inhaled antibiotics and long-term use of oxygen were associated with worse survival. Contrary to previous literature [5], no difference in survival was noted between males and females or in those with CFRD. NICK et al. [16] studied individuals who survived to $\geqslant 40$ years of age, a survivors group. Patients diagnosed in adulthood had milder clinical phenotypes than those diagnosed before the age of 10 years, and a female-associated survival disadvantage persisted in those diagnosed in childhood, whereas this did not exist in the adult-diagnosed cohort.

Accurately predicting life expectancy in $\mathrm{CF}$ is vital to allow CF caregivers to: 1) intervene to change modifiable risk factors; 2) intensify therapy for high-risk groups; 3) provide clinicians with a method to quantify survival that can guide decisions related to patient care; and 4) allow physicians and caregivers to explain the risks and benefits of certain interventions better.

The objectives of this study were to identify risk factors associated with survival using contemporary population-based data across the spectrum of disease severity and to identify those subgroups of CF patients at highest risk for death.

\section{Materials and methods}

This population-based cohort study used Canadian CF Registry (CCFR) data to evaluate survival in Canadians with CF between 1990 and 2012.

\section{Data sources}

The CCFR contains detailed demographic and annual clinical information on CF patients receiving clinical care at the 43 accredited CF centres across Canada. Nutritional markers and lung function are recorded from the first stable clinic visit of the year, while other variables, such as sputum bacteriology, CF-related complications, pancreatic status and hospitalisations, reflect events that occurred throughout each calendar year. Registry data undergo routine validation checks to ensure that they are free of errors, and discrepancies are resolved by cross-referencing with original sources at the reporting CF centre. In 2013, all CF clinics were provided with a list of transplanted individuals from their centre who were recorded as alive to confirm their vital statistics as of December 31, 2012 [17]. Newborn screening (NBS) programmes were implemented in five out of 10 provinces in the latter part of the study period (Alberta: 2007; Ontario: 2008; Saskatchewan: 2009; British Columbia: 2009; Manitoba: 2011); therefore, given the variable inclusion of NBS programmes across the country and the small number of patients affected, we did not examine the impact of NBS on survival in this study.

All individuals within the registry provided informed consent to have their data collected and be used for research purposes. The study was approved by the Research Ethics Board at St Michael's Hospital, Toronto, Canada.

\section{Statistical analysis}

For the characteristics of the CF population followed between 1990 and 2012, simple descriptive statistics (mean, median and proportions) were used. Median age at death per year and death rate (deaths per year/ total number of recorded patients in that year; deaths per year/Canadian population) were calculated as well as the annual proportion of new CF cases. Trends over time were evaluated using nonparametric tests for trend and linear regression analysis. 
Changes in survival over time

Using data from 1990-2012, median age of survival (including 95\% CI) was calculated based on a moving 5 -year window, and calculated using Cox proportional hazards models in the overall cohort and within the following categories: sex, homozygous for the $\triangle F 508$ mutation of the CFTR gene and age of diagnosis.

\section{Contemporary predictors of survival}

Kaplan-Meier survival curves were computed to compare survival between the sexes, regions and Burkholderia cepacia complex (BCC) status. Cox proportional hazards models were used to estimate the effects of multiple demographic and clinical variables on mortality for individuals followed between 2000 and 2012. Data were censored in the year of the first transplant or the last reporting year if the subject did not receive a transplant. We censored on the first transplant date because the impact of clinical variables in the setting of transplant do not reflect CF-lung disease. The proportional hazards assumption was tested for each factor. All factors found to be marginally significant $(p<0.15)$ in univariable testing or those with strong a priori hypotheses were added to a multivariable model using a step-wise approach. The impact of sex and region were evaluated separately, testing for interactions between all variables. Confounding was assessed by evaluating whether the hazard ratio (HR) for region changed by $>10 \%$ with the addition of each variable.

\section{Risk factors}

Time independent

Basic demographic factors, such as sex, age at diagnosis and genotype, were considered time-independent variables. Genotype was classified as homozygous for the $\Delta \mathrm{F} 508$ mutation, heterozygous for $\Delta \mathrm{F} 508$, other or missing. Because of the close genotype-phenotype relationship between genotype and pancreatic status, pancreatic status was used as a surrogate in the multivariable analysis in order to reduce the loss of subjects due to missing genotype data. Individuals were categorised into one of four regions based on the reporting centre, where the majority of the clinic visits occurred: West (all provinces west of Ontario), Ontario, Quebec and East (all provinces east of Quebec). Age at diagnosis was categorised as <2, 2-18 or $>18$ years. Pancreatic-insufficient or pancreatic-sufficient classification was based on enzyme usage.

\section{Time dependent}

The registry records clinical data (i.e. height, weight and lung function) from the first stable visit of each reporting year. FEV1 was expressed as a percentage of the normal predicted values for height, age and sex $[18,19]$. Body mass index (BMI) was calculated using weight/height ${ }^{2}$. BMI percentiles were calculated for children between the ages of 2 and 18 years using Centers for Disease Control growth charts [20]. Children were then classified into three groups based on the BMI percentile: underweight (BMI percentile $\leqslant 12 \%$ ), adequate weight (BMI percentile between $13 \%$ and $84 \%$ ) and overweight (BMI percentile $\geqslant 85 \%$ ). For individuals $\geqslant 18$ years of age, subjects were classified into one of three BMI categories based on World Health Organization guidelines [21]: underweight $\left(<18.5 \mathrm{~kg} \cdot \mathrm{m}^{-2}\right)$, adequate weight $\left(18.5-24.9 \mathrm{~kg} \cdot \mathrm{m}^{-2}\right)$ and overweight $\left(\geqslant 25.0 \mathrm{~kg} \cdot \mathrm{m}^{-2}\right)$. CFRD was defined as hyperglycaemia based on oral glucose tolerance test results or random blood sugar measurements requiring insulin therapy [22]. For sputum bacteriology, clinics reported any positive culture in the year for the following bacteria: BCC, P. aeruginosa, Staphylococcus aureus, Stenotrophomonas maltophilia and methicillin-resistant S. aureus (MRSA). The annual number of pulmonary exacerbations requiring either a hospitalisation or the initiation of home intravenous antibiotic therapy was calculated, and categorised as $0,1-2$ or $\geqslant 3$.

\section{Results}

Cohort description: 1990-2012

There were 5787 individuals with CF followed in the registry between 1990 and 2012 (fig. 1). In 2012, there were 29 new cases of CF per 100000 Canadian births, which represent a rate of one in 3400 births. Over the 20-year period, 5.6\% (322 out of 5787) of subjects were considered lost to follow-up (LTFU) and, of those, $96.9 \%$ were pre-transplant. The majority of subjects were followed annually (97.8\%); however, $1.7 \%$ of patients were missing for $1-4$ years before returning to a CF centre and $0.31 \%$ returned after $\geqslant 5$ years of nonattendance. There were no sex differences in the proportion of patients LTFU $(p=0.76)$; however, the greatest proportion of those LTFU were from Ontario (8.1\%), compared with 6.0\% from the West, 5.4\% from Quebec and 6.5\% from the East.

\section{Changes in survival and clinical characteristics: 1990-2012}

Canadian median age of survival increased from 31.9 years (95\% CI 28.3-35.2 years) in 1990 to 49.7 years (95\% CI 46.1-52.2 years) in 2012 (fig. 2). The proportion of subjects who were homozygous for $\Delta$ F508 ( $49.6 \%$ in 1990 to $48.9 \%$ in 2012 , test for trend $\mathrm{p}=0.05$ ) or pancreatic insufficient $(88.6 \%$ in 1990 to $86.6 \%$ in 2012 , test for trend $\mathrm{p}<0.001)$ decreased, while that of those with the $\mathrm{R} 117 \mathrm{H}$ genotype increased $(0.61 \%$ 


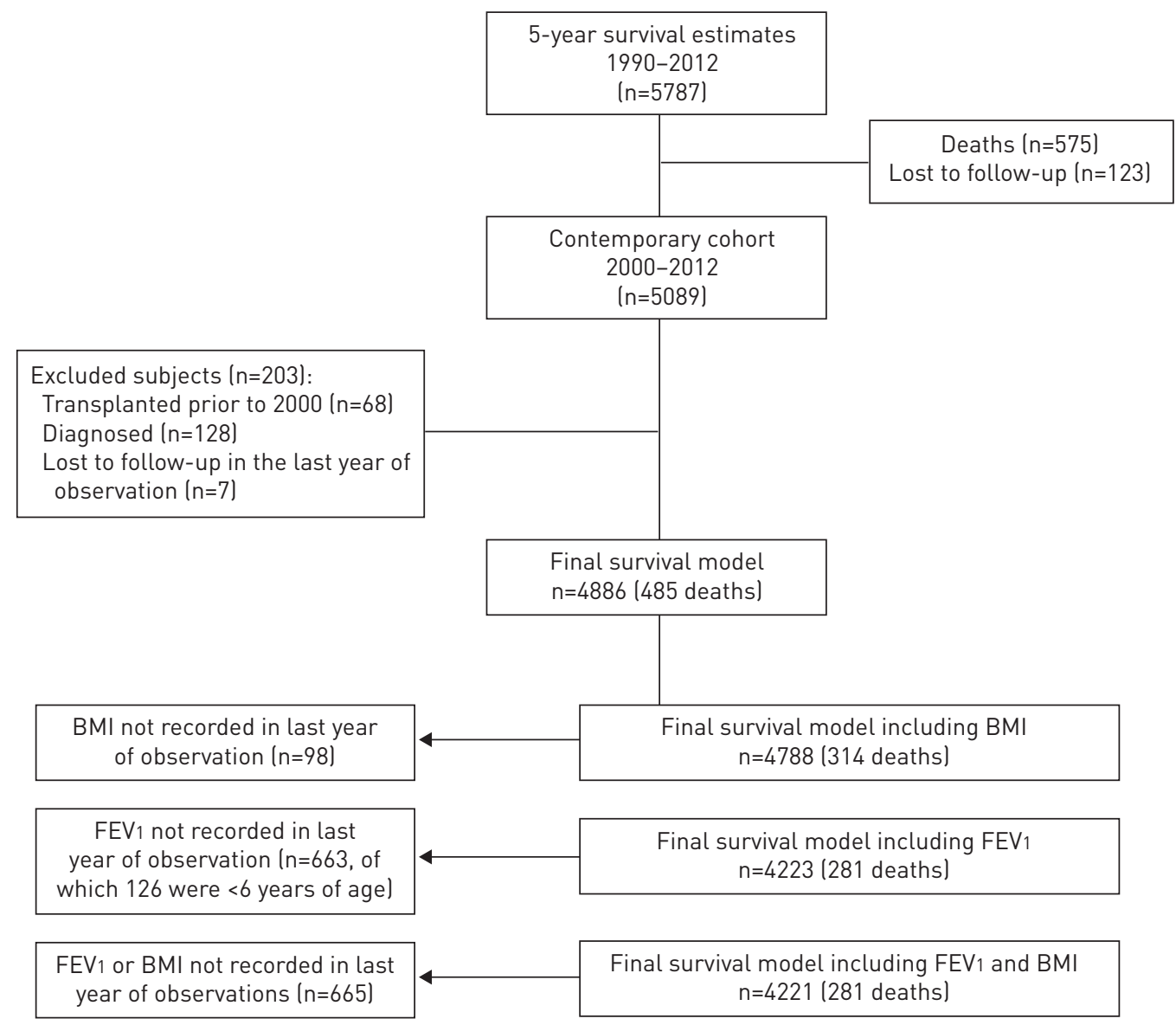

FIGURE 1 Study cohort creation flow diagram. BMI: body mass index; FEV1: forced expiratory volume in $1 \mathrm{~s}$.

in 1990 to $1.9 \%$ in 2012 , test for trend $\mathrm{p}<0.001$ ) over time, suggesting that milder clinical phenotypes are captured in the registry over the study period. However, we found a similar increase in median survival age when the cohort was restricted to 1) individuals who are homozygous for the $\Delta$ F508 mutation, 2) individuals diagnosed before the age of 2 years and 3) individuals diagnosed before the age of 18 years, suggesting the increase in survival is not solely due to the inclusion of milder cases in the registry (fig. 3). The annual median age at death increased from 21.7 years in 1990 to 32.0 years in 2012 ( $p=0.003$ ), while the death rate per year declined from 1.4 deaths per person in 1990 to 0.99 deaths per person in 2012 $(\mathrm{p}<0.001)$ (figs 4 and 5).

FIGURE 2 Median age of survival using a 5-year moving window, overall and by sex.

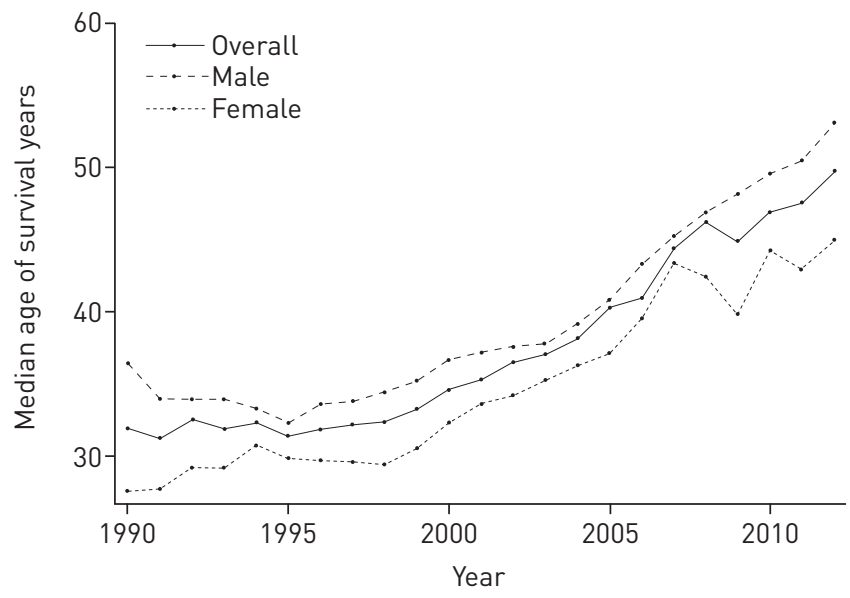




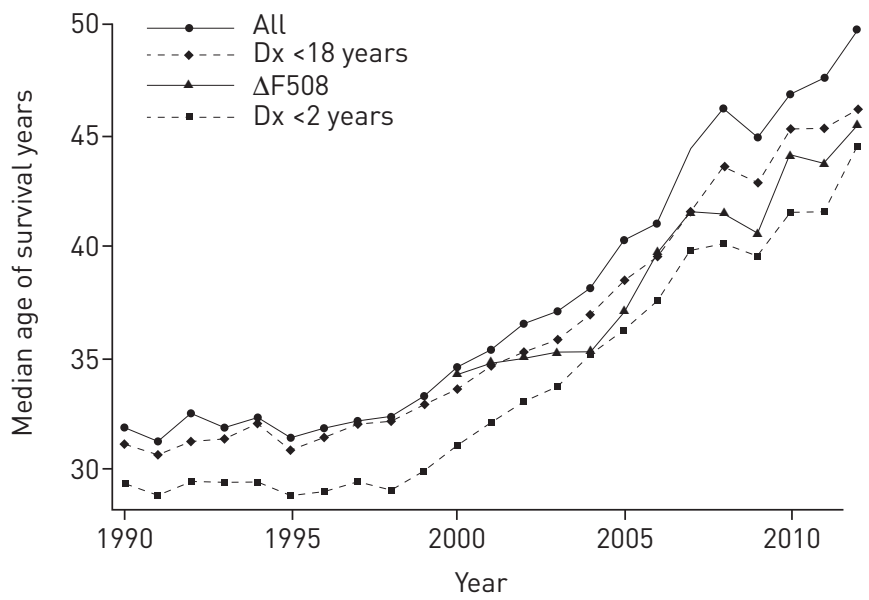

FIGURE 3 Median age of survival by genotype and age at diagnosis. Median survival for those who were homozygous for $\Delta \mathrm{F} 508$ begins in 2000 , as complete genotype data were available in the Canadian registry from 2000 onwards. Dx: age at diagnosis.

Our data show there were fewer malnourished and more overweight Canadians living with CF in our cohort. In 1990, 31\% of individuals in the registry were considered underweight, which decreased to $19 \%$ in 2012 (slope $-0.55 \%$ per year, $\mathrm{p}<0.001$ ). The proportion of overweight individuals increased from $8 \%$ in 1990 to $16 \%$ in 2012 (slope $0.37 \%$ per year, $\mathrm{p}<0.001$ ). The annual number of transplants recorded in the registry also increased over the study period $(\mathrm{p}<0.001)$. The median FEV1 increased over time from $71.4 \%$ predicted in 1990 to $75.4 \%$ predicted in 2012 (slope $0.06 \%$ per year, $\mathrm{p}=0.04$ ). The increase in FEV1 is also increasing over time in the context of the median age of the CF population, going from 12.9 years in 1990 to 20.1 years in $2012(\mathrm{p}<0.001)$. The prevalence of $P$. aeruginosa, S. aureus, MRSA and S. maltophilia infection increased over the study period, while that of BCC decreased (data not shown).

Both the number of newly diagnosed cases and the proportion of individuals diagnosed in adulthood remained stable over the study period $(\mathrm{p}>0.05)$.

\section{Contemporary predictors of survival: 2000-2012}

There were 5089 individuals with CF followed in the registry between 2000 and 2012 (fig. 1). A total of 4886 patients contributed to the contemporary cohort analysis, during which time 485 patients died. Table 1 summarises the demographic and clinical characteristics based on their last recorded measurement.

The univariable and multivariable proportional hazards analyses are shown in table 2. The annual rate of pulmonary exacerbations was the strongest variable associated with risk of death (HR 4.53 for $\geqslant 3$ exacerbations per year, $\mathrm{p}<0.0001$; HR 2.98 for $1-2$ exacerbations/year, $\mathrm{p}<0.001)$. Malnutrition was associated with an increased risk of death, with a HR of 2.12 compared with those with adequate nutritional status. Infection with S. maltophilia showed a protective effect (HR 0.75, p=0.03). FEV1 remained an independent risk factor (higher FEV1 was associated with a lower risk of death); however, a significant interaction was found between pulmonary exacerbations and FEV1 $(p<0.001)$. Individuals with

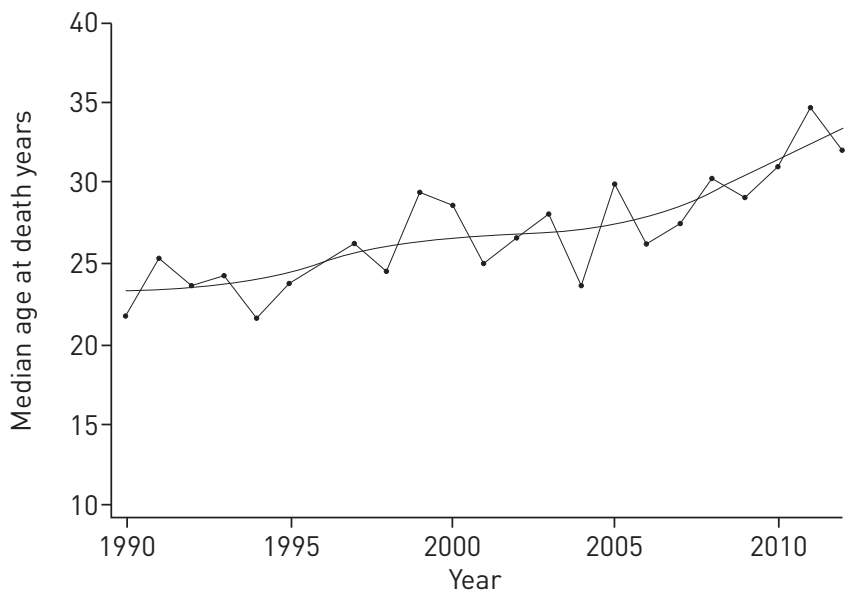

FIGURE 4 Median age at death over time. 
FIGURE 5 Annual death rate, calculated by taking the number of deaths in a year divided by the total number of patients reported on in that year.

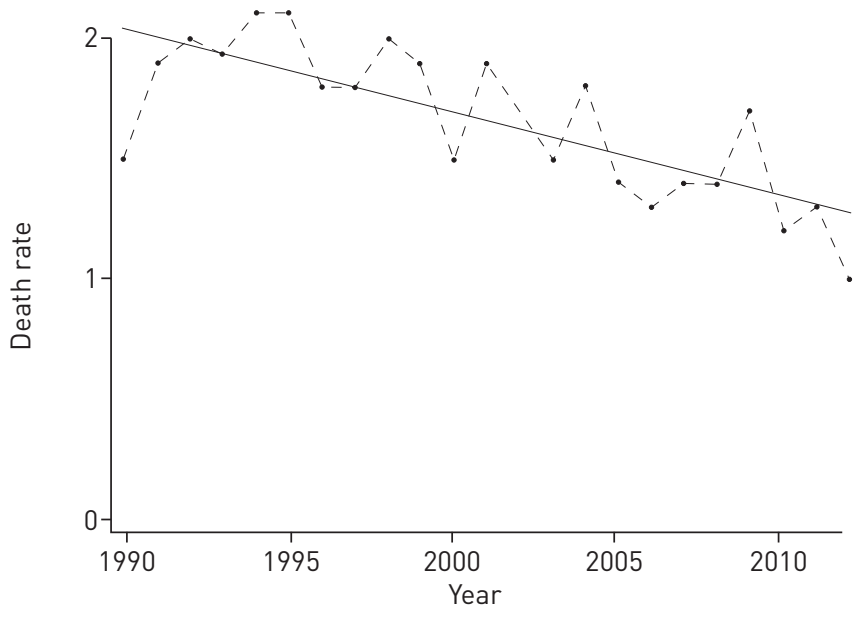

lower lung function were more likely to have pulmonary exacerbations than those with higher lung function.

\section{Sex gap}

In the multivariable model, the adjusted risk of death in females was higher than in males (HR 1.28, $\mathrm{p}=0.05$ ). A significant interaction was identified between sex and CFRD. Among individuals with CFRD, the HR for death was 1.81 ( $\mathrm{p}=0.004)$ in females compared with males. Among males, the risk of death was similar regardless of whether or not CFRD was present (HR 0.79, $\mathrm{p}=0.23$ ). When the sex-CFRD interaction was included in the multivariable model, sex was no longer significant (HR 1.02, p=0.89), suggesting that the differential negative effect of having CFRD in females may explain much of the sex gap in CF survival. Within the contemporary cohort, significantly more females had CFRD compared with males ( $30 \%$ versus $17 \%$, respectively; $\mathrm{p}<0.0001)$.

\section{Regional differences in survival}

Based on our univariable analysis, regional variations in survival were found; however, after adjusting for exacerbation frequency, BCC and FEV1, region was no longer an independent predictor of death.

\section{Transplant}

In the multivariable model, subjects were censored at transplant, as the interpretation of clinical variables post-transplant differs from pre-transplant CF disease. In order to illustrate the impact of including post-transplant deaths on survival estimates, Kaplan-Meier survival curves were calculated in two ways: 1) censoring at the time of transplant and 2) including deaths post-transplant (fig. 6). Censoring subjects at the time of transplant overestimated the overall survival estimate.

\section{Discussion}

Our study showed that Canadian CF median survival age and age at death have increased while the annual death rate has decreased over the last 20 years. Similar increases in survival were seen when the analysis was restricted to those with severe phenotypes, suggesting that the improvements were not solely driven by the inclusion of milder cases in the registry. Canadians with CF have better nutrition and improved lung function than in previous decades, which has probably contributed to improved overall survival. In addition, the prevalence of bacteria such as $S$. maltophilia and MRSA has increased while the prevalence of BCC is declining. These trends are important to recognise as these data have potential infection control implications and prior literature has shown that infection with these organisms can have a significant negative impact on survival $[23,24]$.

The results of our study confirm that frequent pulmonary exacerbations are associated with increased mortality, which is consistent with the previous literature [25]. This finding is not surprising, as many CF deaths are preceded by worsening infection; however, minimising these events is critical, as the rate of decline in FEV1 is greatest when exacerbations occurs frequently and within a short time frame [26]. Malnutrition continues to be an important risk factor for death. Although there were fewer malnourished patients in recent decades, $19 \%$ of individuals in 2012 had a BMI $<19 \mathrm{~kg} \cdot \mathrm{m}^{-2}$, which highlights a population who could benefit from aggressive nutritional support. By quantifying the survival risk associated with clinical characteristics, healthcare providers and patients can better understand the 
TABLE 1 Descriptive characteristics of study cohort: $2000-2012^{\#}$

\begin{tabular}{|c|c|}
\hline Subjects $\mathbf{n}$ & 4886 \\
\hline Newly diagnosed cases $\mathrm{n}$ & 1498 \\
\hline Females & 46.9 \\
\hline Caucasians & 92.8 \\
\hline Age at last visit years & $22.0(0-79.3)$ \\
\hline Age at diagnosis years median & 0.5 \\
\hline \multicolumn{2}{|l|}{ Age at diagnosis } \\
\hline $0-2$ years & 66.8 \\
\hline $2-18$ years & 26.2 \\
\hline$>18$ years & 6.9 \\
\hline \multicolumn{2}{|l|}{ Genotype } \\
\hline Homozygous $\Delta \mathrm{F} 508$ & 48.1 \\
\hline Heterozygous $\Delta \mathrm{F} 508$ & 38.1 \\
\hline Other & 9.9 \\
\hline Missing & 4.0 \\
\hline FEV $1 \%$ predicted & $67.7(9.5-145.1)$ \\
\hline \multicolumn{2}{|l|}{ BMI category } \\
\hline Normal weight & 59.0 \\
\hline Overweight & 13.4 \\
\hline Underweight & 17.9 \\
\hline Missing & 9.8 \\
\hline \multicolumn{2}{|l|}{ Microbiology } \\
\hline Pseudomonas aeruginosa & 79.3 \\
\hline Staphylococcus aureus & 87.8 \\
\hline Stenotrophomonas maltophilia & 37.8 \\
\hline Burkholderia cepacia complex & 11.6 \\
\hline MRSA & 9.0 \\
\hline Pancreatic insufficiency & 85.9 \\
\hline CF-related diabetes & 24.1 \\
\hline \multicolumn{2}{|l|}{ Region $^{+}$} \\
\hline Quebec & 29.1 \\
\hline East & 9.1 \\
\hline Ontario & 34.5 \\
\hline West & 27.3 \\
\hline \multicolumn{2}{|l|}{ Exacerbations per year } \\
\hline 0 & 68.6 \\
\hline $1-2$ & 21.2 \\
\hline$\geqslant 3$ & 10.2 \\
\hline Transplants & 472 (9.7) \\
\hline Males & 255 \\
\hline Females & 217 \\
\hline Total deaths $\mathrm{n}$ & 485 \\
\hline Males & $227(46.6)$ \\
\hline Females & $260(53.4)$ \\
\hline
\end{tabular}

Data are presented as \%, median (range) or $\mathrm{n}(\%)$, unless otherwise stated. FEV1: forced expiratory volume in $1 \mathrm{~s}$; BMI: body mass index; MRSA: methicillin-resistant S. aureus; CF: cystic fibrosis. \#: last recorded measurement used to summarise the data; " : reflects the proportion of individuals who ever had a positive sputum culture in the study period; ${ }^{+}$: based on the region in which the subject spent the longest time.

implications of untreated conditions such as malnutrition and recurrent exacerbations, which may influence future clinical decisions. Individuals may be motivated to accept therapy that has been shown in previous studies to reduce exacerbation rates or improve nutrition if they were cognisant of the significant impact these factors had on survival. In addition, nonmodifiable factors that increase the risk of death are important to characterise, as these may identify high-risk groups, such as females with CFRD, who could benefit from intensive monitoring to maintain their health.

Poorer female survival in CF has been reported in several countries $[8,27,28]$. Our study suggested a sex gap in survival; however, the risk of death in females was of borderline statistical significance once we adjusted for multiple covariates. Recent literature suggests the previously seen sex gap in survival in CF is disappearing. NICK et al. [16] did not see an effect of sex on survival in individuals diagnosed in childhood and, in fact, there was a survival advantage observed for women diagnosed in adulthood. Furthermore, in 
TABLE 2 Cox proportional hazard models for death in the contemporary cystic fibrosis (CF) cohort: 2000-2012

Sex
Male
Female
Genotype
Other
Homozygous $\Delta$ F508
Heterozygous $\Delta$ F508
Missing
Pancreatic insufficiency
Sufficient
Insufficient
Age at diagnosis
$<2$ years
2-18 years
>18 years
Longest time in a region
Quebec
East
Ontario
West
Exacerbations per year
0
1-2
₹3
CF-related diabetes
Microbiology
Burkholderia cepacia complex
Staphylococcus aureus
Pseudomonas aeruginosa
Stenotrophomonas maltophilia
MRSA
BMI categories
Adequate weight
Overweight
Underweight
FEV1\% predicted
Birth year

Univariate

\section{Multivariate}

Ref.

$1.35(1.12-1.61)$

Ref.

$1.28(1.00-1.63)$

Ref.

$1.54(1.08-2.21)$

$1.20(0.84-1.73)$

$3.01(1.89-4.81)$

Ref.

$3.18(2.19-4.61)$

Ref.

$0.62(0.50-0.77)$

$0.30(0.20-0.44)$

Ref.

$1.53(1.13-2.09)$

$1.01(0.81-1.28)$

$1.27(1.00-1.61)$

Ref.

$6.17(3.69-10.31)$

$11.12(5.58-21.78)$

$2.26(1.36-3.78)$

$2.51(1.40-4.50)$

$0.32(0.19-0.53)$

$2.05(0.98-4.27)$

$1.17(0.73-1.86)$

$0.70(0.25-1.94)$

Ref.

$0.22(0.06-0.80)$

$12.44(6.26-24.75)$

$0.91(0.89-0.93)$

$0.96(0.93-0.99)$
Ref.

$2.19(1.15-4.17)$

Ref.

$0.79(0.59-1.06)$

$0.55(0.28-1.11)$

Ref.

$2.98(2.14-4.15)$

4.53 (3.19-6.43)

$1.89(1.44-2.49)$

$0.75(0.58-0.97)$

Ref.

$0.80(0.47-1.35)$

$2.12(1.60-2.82)$

$0.95(0.94-0.96)$

Data are presented as hazard ratio $(95 \%$ CI). MRSA: methicillin-resistant S. aureus; BMI: body mass index; FEV1: forced expiratory volume in $1 \mathrm{~s} .{ }^{*}$ : reference group for each bacterium was individuals who were culture negative for that bacterium.

FIGURE 6 Kaplan-Meier survival curves illustrating the effect of including post-transplant deaths versus censoring at transplantation.

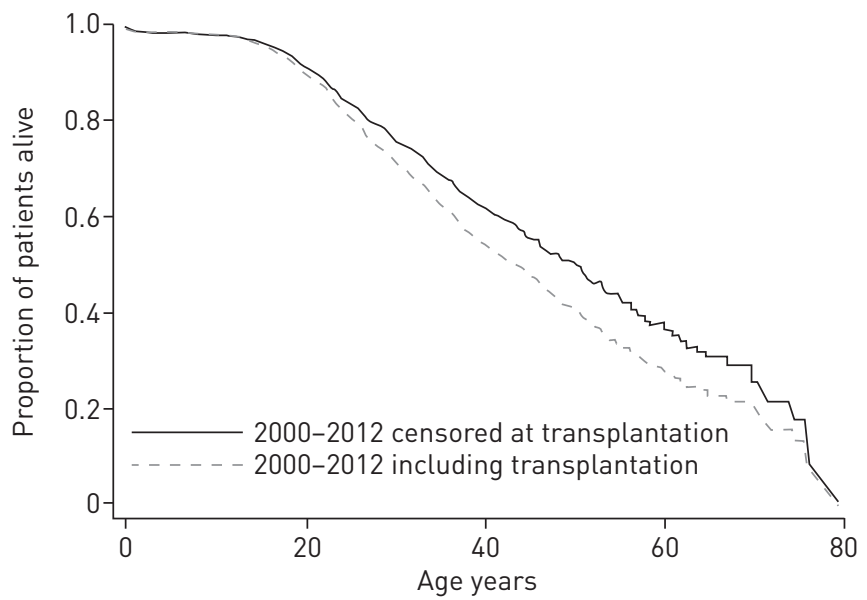


those with severe lung disease, sex was not a significant predictor of survival [15]. Even more dramatically, our analysis suggests that a CFRD-sex interaction at least partly explained the observed sex gap in survival. Earlier work by MiLla et al. [5] also identified worse survival in females with CFRD; however, a subsequent paper found that the sex difference in mortality in those with CFRD had disappeared [29]. These authors suggested that early diagnosis and more aggressive treatment of CFRD may be the reason for this finding. However, further confirmatory research is necessary to conclude that the differential survival in males and females is driven by worse survival in women with CFRD. In addition, the median survival age in males has been steadily increasing, whereas female median survival age has been variable, particularly between 2007 and 2012. Our analyses did not identify a single cause for this variability, which is probably multifactorial. One could hypothesise that hormonal influences may be playing a role as the female CF population ages; however, this needs to be systematically investigated in future studies.

Despite Canada's universal health coverage, there are regional differences in healthcare policies, access to treatments and care. Identifying regional differences in health outcomes can elucidate potential gaps in healthcare delivery. COREY et al. [28] reported regional differences in Canadian CF survival using registry data from 1985-1989. Our results showed that regional differences in CF survival were explained by patient characteristics, some of which were not available at the time of the earlier paper. Although there may be differences in certain clinical outcomes across Canada, region itself did not predict survival in a contemporary cohort of individuals with CF.

Lung transplantation can be a life-saving therapy for those with end-stage lung disease; thus, survival statistics should include transplanted individuals when possible. Transplant is an important contributor to survival estimates, which are affected by statistical methodology (i.e. whether or not patients are censored at transplant) and by the rigour with which vital statistics are captured post-transplant. We illustrated that survival estimates differ depending on whether or not post-transplant deaths are included in the analysis. Post-transplant patients may not be followed as closely by CF centres; therefore, this group is at high risk for being LTFU in national CF registries. This is important to recognise for all national registries that produce CF survival statistics and extra effort should be placed on determining the vital status of individuals, particularly post-transplantation, so that accurate survival estimates can be calculated.

The strengths of our study include the large sample size, the longitudinal and comprehensive data within the CCFR, and the standardised way data were captured using clear definitions. The registry has few patients LTFU and complete data on patient deaths, making this a robust resource for epidemiology studies. Our results showed a protective effect associated with $S$. maltophilia, which differs from a previous study by WATERS et al. [23], who reported that chronic infection with S. maltophilia was associated with increased mortality or lung transplantation. The discrepancy in results may be due to our inability to differentiate between chronic and intermittent infection, which is a limitation of the data source. The registry captures lung function and nutritional markers once per year, which may not reflect the true variability of these measurements; however, we used longitudinal data over decades to capture variability over time. Finally, as many subjects had missing genotype data, we used pancreatic status as a surrogate marker given the close genotype-phenotype relationship between these two variables. Given the large sample size and wide spectrum of disease captured, our results would apply to the overall CF population; however, variations in healthcare delivery and variable definitions may differ between countries, limiting generalisability.

In conclusion, life expectancy in Canadians with CF is increasing; patients are better nourished and lung function has improved over time. Despite these improvements, suboptimal nutrition and recurrent pulmonary exacerbations occur and remain important predictors of death. The sex gap in CF survival appears to be explained by an increased prevalence of CFRD in females and increased mortality in women with CFRD. However, further studies are needed to better understand the sex-CFRD interaction.

\section{References}

1 Dodge JA, Lewis PA, Stanton M, et al. Cystic fibrosis mortality and survival in the UK: 1947-2003. Eur Respir J 2007; 29: 522-526.

2 Slieker MG, Uiterwaal CSPM, Sinaasappel M, et al. Birth prevalence and survival in cystic fibrosis. Chest 2005; 128: 2309-2315.

3 Kerem E, Reisman J, Corey M, et al. Prediction of mortality in patients with cystic fibrosis. N Engl J Med 1992; 326: $1187-1191$.

4 Milla CE, Warwick WJ. Risk of death in cystic fibrosis patients with severely compromised lung function. Chest 1998; 113: 1230-1234.

5 Milla CE, Billings J, Moran A. Diabetes is associated with dramatically decreased survival in female but not male subjects with cystic fibrosis. Diabetes Care 2005; 28: 2141-2144.

6 Aurora P, Wade A, Whitmore P, et al. A model for predicting life expectancy of children with cystic fibrosis. Eur Respir J 2000; 16: 1056-1060. 
7 Kulich M, Rosenfeld M, Goss $\mathrm{CH}$, et al. Improved survival among young patients with cystic fibrosis. J Pediatr 2003; 142: 631-636.

8 Rosenfeld M, Davis R, FitzSimmons S, et al. Gender gap in cystic fibrosis mortality. Am J Epidemiol 1997; 145: 794-803.

9 Liou TG, Adler FR, FitzSimmons SC, et al. Predictive 5-year survivorship model of cystic fibrosis. Am J Epidemiol 2001; 153: 345-352.

10 Ramsey BW, Davies J, McElvaney NG, et al. A CFTR potentiator in patients with cystic fibrosis and the G551D mutation. N Engl J Med 2011; 365: 1663-1672.

11 Fuchs HJ, Borowitz DS, Christiansen DH, et al. Effect of aerosolized recombinant human DNase on exacerbations of respiratory symptoms and on pulmonary function in patients with cystic fibrosis. N Engl J Med 1994; 331: 672-673.

12 Ramsey BW, Pepe MS, Quan JM, et al. Intermittent administration of inhaled tobramycin in patients with cystic fibrosis. N Engl J Med 1999; 340: 23-30.

13 Liou TG, Adler FR, Cahill BC, et al. Survival effect of lung transplantation among patients with cystic fibrosis. JAMA 2001; 286: 2683-2689.

14 Moran A, Becker D, Casella SJ, et al. Epidemiology, pathophysiology, and prognostic implications of cystic fibrosis-related diabetes: a technical review. Diabetes Care 2010; 33: 2677-2683.

15 George PM, Banya W, Pareek N, et al. Improved survival at low lung function in cystic fibrosis: cohort study from 1990 to 2007. BMJ 2011; 342: d1008.

16 Nick JA, Chacon CS, Brayshaw SJ, et al. Effects of gender and age at diagnosis on disease progression in long-term survivors of cystic fibrosis. Am J Respir Crit Care Med 2010; 182: 614-626.

17 Cystic Fibrosis Canada. Annual Registry Report. Toronto, Cystic Fibrosis Canada, 2011.

18 Hankinson JL, Odencrantz JR, Fedan KB. Spirometric reference values from a sample of the general U.S. population. Am J Respir Crit Care Med 1999; 159: 179-187.

19 Wang X, Dockery DW, Wypij D, et al. Pulmonary function between 6 and 18 years of age. Pediatr Pulmonol 1993; 15: 75-88.

20 Kuczmarski RJ, Ogden CL, Grummer-Strawn LM, et al. CDC growth charts: United States. Adv Data 2000; 314: $1-27$.

21 World Health Organization. BMI Classification. Geneva, WHO, 2013.

22 Moran A, Hardin D, Rodman D, et al. Diagnosis, screening and management of cystic fibrosis related diabetes mellitus: a consensus conference report. Diabetes Res Clin Pract 1999; 45: 61-73.

23 Waters V, Atenafu EG, Lu A, et al. Chronic Stenotrophomonas maltophilia infection and mortality or lung transplantation in cystic fibrosis patients. J Cyst Fibros 2013; 12: 482-486.

24 Dasenbrook EC, Checkley W, Merlo CA, et al. Association between respiratory tract methicillin-resistant Staphylococcus aureus and survival in cystic fibrosis. JAMA 2010; 303: 2386-2392.

25 de Boer K, Vandemheen KL, Tullis E, et al. Exacerbation frequency and clinical outcomes in adult patients with cystic fibrosis. Thorax 2011; 66: 680-685.

26 Waters V, Stanojevic S, Atenafu EG, et al. Effect of pulmonary exacerbations on long-term lung function decline in cystic fibrosis. Eur Respir J 2012; 40: 61-66.

27 Davis PB. The gender gap in cystic fibrosis survival. J Gend Specif Med 1999; 2: 47-51

28 Corey M, Farewell V. Determinants of mortality from cystic fibrosis in Canada, 1970-1989. Am J Epidemiol 1996 143: 1007-1017.

29 Moran A, Dunitz J, Nathan B, et al. Cystic fibrosis-related diabetes: current trends in prevalence, incidence, and mortality. Diabetes Care 2009; 32: 1626-1631. 